\title{
Serum biomarkers as predictors of long-term outcome in severe traumatic brain injury: analysis from a randomized placebo-controlled Phase II clinical trial
}

\author{
Amol Raheja, MCh, ${ }^{1}$ Sumit Sinha, MCh, ${ }^{1}$ Neha Samson, MTech, ${ }^{1}$ Sanjeev Bhoi, MD, ${ }^{2}$ \\ Arulselvi Subramanian, MD, ${ }^{3}$ Pushpa Sharma, $\mathrm{PhD},{ }^{4}$ and Bhawani Shankar Sharma, $\mathrm{MCh}^{1}$ \\ Departments of ${ }^{1}$ Neurosurgery, ${ }^{2}$ Emergency Medicine, and ${ }^{3}$ Laboratory Medicine, All India Institute of Medical Sciences, New \\ Delhi, India; and ${ }^{4}$ Department of Anaesthesiology, Uniformed Services University of the Health Sciences, Bethesda, Maryland
}

OBJECTIVE There has been increased interest in the potential importance of biochemical parameters as predictors of outcome in severe traumatic brain injury (STBI).

METHODS Of 107 patients with sTBI (age 18-65 years with a Glasgow Coma Scale score of 4-8 presenting within 8 hours after injury) who were randomized for a placebo-controlled Phase II trial of progesterone with or without hypothermia, the authors serially analyzed serum biomarkers (S100-B, glial fibrillary acidic protein [GFAP], neuron-specific enolase [NSE], tumor necrosis factor- $\alpha$, interleukin-6 [L-6], estrogen [Eg], and progesterone [Pg]). This analysis was performed using the sandwich enzyme-linked immunosorbent assay technique at admission and 7 days later for 86 patients, irrespective of assigned group. The long-term predictive values of serum biomarkers for dichotomized Glasgow Outcome Scale (GOS) score, functional independence measure, and survival status at 6 and 12 months were analyzed using an adjusted binary logistic regression model and receiver operating characteristic curve.

RESULTS A favorable GOS score (4-5) at 1 year was predicted by higher admission IL-6 (above $108.36 \mathrm{pg} / \mathrm{ml}$; area under the curve [AUC] 0.69 , sensitivity 52\%, and specificity $78.6 \%$ ) and Day $7 \mathrm{Pg}$ levels (above $3.15 \mathrm{ng} / \mathrm{ml}$; AUC 0.79, sensitivity $70 \%$, and specificity $92.9 \%$ ). An unfavorable GOS score (1-3) at 1 year was predicted by higher Day 7 GFAP levels (above $9.50 \mathrm{ng} / \mathrm{ml}$; AUC 0.82 , sensitivity $78.6 \%$, and specificity $82.4 \%$ ). Survivors at 1 year had significantly higher Day 7 Pg levels (above $3.15 \mathrm{ng} / \mathrm{ml}$; AUC 0.78 , sensitivity $66.7 \%$, and specificity $90.9 \%$ ). Nonsurvivors at 1 year had significantly higher Day 7 GFAP serum levels (above $11.14 \mathrm{ng} / \mathrm{ml}$; AUC 0.81 , sensitivity $81.8 \%$, and specificity $88.9 \%$ ) and Day 7 IL-6 serum levels (above $71.26 \mathrm{pg} / \mathrm{ml}$; AUC 0.87 , sensitivity $81.8 \%$, and specificity $87 \%$ ). In multivariate logistic regression analysis, independent predictors of outcome at 1 year were serum levels of Day $7 \mathrm{Pg}$ (favorable GOS-OR $3.24, \mathrm{Cl} 1.5-7, p=0.003$; and favorable survival-OR 2, Cl 1.2-3.5, $p=0.01$ ); admission IL-6 (favorable GOS-OR 1.04, Cl 1.00-1.08, $p=0.04$ ); and Day 7 GFAP (unfavorable GOS-OR 0.79, $\mathrm{Cl} 0.65-0.95, \mathrm{p}=0.01$; and unfavorable survival-OR 0.80, Cl 0.66-0.96, $p=0.01$.

CONCLUSIONS Serial Pg, GFAP, and IL-6 monitoring could aid in prognosticating outcomes in patients with acute STBI. A cause and effect relationship or a mere association of these biomarkers to outcome needs to be further studied for better understanding of the pathophysiology of sTBI and for choosing potential therapeutic targets.

Clinical trial registration no.: CTRI/2009/091/000893 (http://www.ctri.nic.in).

http://thejns.org/doi/abs/10.3171/2015.6.JNS15674

KEY WORDS glial fibrillary acidic protein; interleukin-6; progesterone; predictors of outcome; serum biomarkers; severe traumatic brain injury; trauma

ABBREVIATIONS AUC = area under the curve; $\mathrm{BBB}=$ blood-brain barrier; $\mathrm{DAI}=$ diffuse axonal injury; $\mathrm{Eg}=$ estrogen; FIM = functional independence measure; $\mathrm{GCS}=$ Glasgow Coma Scale; GFAP = glial fibrillary acidic protein; GOS = Glasgow Outcome Scale; ICP = intracranial pressure; IL = interleukin; IQR = interquartile range; NGF = nerve growth factor; NSE = neuron-specific enolase; $\mathrm{Pg}=$ progesterone; ROC = receiver operating characteristic; sTBI = severe traumatic brain injury; TNF- $\alpha=$ tumor necrosis factor- $\alpha$.

SUBMITTED March 25, 2015. ACCEPTED June 18, 2015.

INCLUDE WHEN CITING Published online January 1, 2016; DOI: 10.3171/2015.6.JNS15674. 
$\mathrm{P}$ ATHOPHYSIOLOGY in traumatic brain injury (TBI) is a complex interplay of brain tissue-specific antigens, hormonal imbalance, and cytokine-mediated humoral and cellular immune reactions. , 29,32 Due to the limitations of existing clinical and radiological evaluations for assessment and prognostication of TBI severity, there has been a considerable paradigm shift toward development and use of biochemical markers to delineate the extent of brain tissue damage and to independently predict global outcome. Neuronal (ubiquitin C-terminal hydrolase L1, neuron-specific enolase [NSE] $)^{4,7,19-21}$ and glial (glial fibrillary acidic protein [GFAP], S100-B) ${ }^{10,18,28,31,37}$ markers have been studied extensively over the past decade in various laboratory and clinical studies, with heterogeneous results. Similarly, the role of proinflammatory (interleukin [IL]-6, IL-1, tumor necrosis factor- $\alpha$ $[\mathrm{TNF}-\alpha]$ ) and antiinflammatory (IL-4, IL-10, transforming growth factor) cytokines has been validated in recent trials. $1,2,6,8,9,11-13,22,26,27,30,36$

More recently, posttraumatic suppression of the hypothalamic-pituitary-adrenal axis and an increase in cytokine-mediated peripheral aromatase activity, leading to an imbalance in sex steroid (estrogen [Eg], progesterone [Pg], and testosterone) serum levels, have been areas of active interest for precise understanding of the mechanisms involved. ${ }^{5,15,23,25,29}$ Most of the recent literature deals with these biomarkers in isolation, and there is a paucity of existing literature for establishing a comprehensive model dealing with all 3 domains together. To this end, we serially analyzed serum biomarkers (S100-B, GFAP, NSE, TNF- $\alpha$, IL-6, Eg, and Pg) as part of a randomized placebo-controlled Phase II trial of progesterone with or without hypothermia in patients with severe TBI (sTBI), and analyzed the long-term predictive value of these biomarkers on Glasgow Outcome Scale (GOS) score, functional independence measure (FIM), and survival status at 6 and 12 months.

\section{Methods \\ Study Design}

This study was part of a prospective, outcome-assessor- and statistician-blinded, randomized, placebo-controlled Phase II trial of progesterone with or without hypothermia (Factorial design, unpublished data [S. Sinha et al., Neurology India, in press; accepted October 2015]). The study population included adult (age 18-65 years) patients with sTBI (Glasgow Coma Scale [GCS] Score 4-8) who presented at our tertiary care trauma center within 8 hours after injury. The study was registered under the Clinical Trials Registry-India (http://www.ctri.nic.in) with trial number CTRI/2009/091/000893. We obtained approval from our institutional ethics committee prior to commencement of the trial. Of 107 patients with sTBI who were randomized for the trial, we serially analyzed 7 serum biomarkers (S100-B, GFAP, NSE, TNF- $\alpha$, IL-6, $\mathrm{Eg}$, and $\mathrm{Pg}$ ) in 86, irrespective of assigned group. We analyzed the long-term predictive value of serum biomarkers on dichotomized GOS score (poor recovery, GOS 1-3; good recovery, GOS 4-5), dichotomized FIM score (functionally dependent, FIM $\leq 108$; functionally independent,
FIM 109-126), and survival status (survivor/nonsurvivor) at 6- and 12-month follow-up.

\section{Biomarker Assessment}

The serum levels of S100-B (BioVendor), NSE (DRG International), IL-6 (Diclone), GFAP (BioVendor), TNF$\alpha$ (Diclone), Pg (DRG), and Eg (DRG) were determined using a commercially available kit based on the principle of the sandwich enzyme-linked immunosorbent assay technique. The samples for the determination of these biomarkers were taken at the time of admission, immediately after randomization ( $<8$ hours after injury), and then 1 week later (approximately 7 days after injury). A standard curve was prepared using standards supplied with the assay (irrespective of biomarkers), and the readings were analyzed using this standard curve.

\section{Statistical Analysis}

Using SPSS software (version 20, IBM Corp.), the baseline parameters, serum biomarkers, and outcome are presented as either number (percentage), mean $( \pm S D)$, or median (interquartile range [IQR]), wherever appropriate. Comparative analysis for admission, Day 7, and average serum biomarkers (stratified according to outcome) was performed using the Wilcoxon rank-sum test. To define independent factors predicting outcome, a binary logistic regression model was used after adjusting for GCS score at admission and treatment group assigned at randomization. The impact of factors on outcome was expressed as the OR $(95 \% \mathrm{CI})$. A receiver operating characteristic (ROC) curve was made for independent factors predicting outcome, along with estimation of area under the curve (AUC), sensitivity, and specificity of optimal cutoff value (calculated by Youden index). In line with current statistical consensus, an AUC of $0.8-0.9$ is considered very good, $0.7-0.8$ is considered adequate, and $<0.7$ is considered poor in terms of accuracy of the test under consideration. A $\mathrm{p}$ value of $<0.05$ was considered significant.

\section{Results}

\section{Demographic Profile}

A total of 86 patients ( $84.9 \%$ of them male) with mean age 32.3 years were analyzed for the current study (Table 1). The average intracranial pressure (ICP) in the present cohort (measured serially for 5 days) was $11.8 \mathrm{~mm} \mathrm{Hg}$. Associated injuries (polytrauma) were present in $62 \%$ of analyzed patients. Contusion (59.3\%) was the most common radiological presentation. Decompressive craniectomy was performed for refractory intracranial hypertension in $32.6 \%$ of patients. The median hospitalization period was 13 days. Sixteen (18.6\%) and 21 (24.4\%) patients were lost to follow-up at 6 and 12 months, respectively. Favorable GOS scores were found in $71.4 \%$ of patients at 6 months and in $78.5 \%$ of patients at 12 months. Also, $63.3 \%$ and $81.5 \%$ of patients were functionally independent at 6 and 12 months, respectively. The mortality rate was $14.3 \%$ and $16.9 \%$ at the 6 - and 12-month follow-up, respectively. 


\section{Comparative Analysis of Serum Biomarkers Stratified by GOS}

The serum levels of Eg, S100-B, and TNF- $\alpha$ were similar across both dichotomized GOS groups at 6 and 12 months ( $p>0.05$ ) (Tables 2 and 3). At 6 and 12 months, the serum levels of Day 7 Pg, NSE levels at admission, and IL-6 levels at admission were significantly higher in favorable outcome groups (Day $7 \mathrm{Pg}: 5.1 \mathrm{ng} / \mathrm{ml}$ vs $2.1 \mathrm{ng} / \mathrm{ml}$ [p $=0.002]$ and $5.1 \mathrm{ng} / \mathrm{ml}$ vs $2 \mathrm{ng} / \mathrm{ml}[\mathrm{p}=0.001]$; admission NSE: $49.2 \mathrm{ng} / \mathrm{ml}$ vs $21.1 \mathrm{ng} / \mathrm{ml}[\mathrm{p}=0.02]$ and $49.2 \mathrm{ng} / \mathrm{ml}$ vs $25.1 \mathrm{ng} / \mathrm{ml}[\mathrm{p}=0.04]$; and admission IL-6: $109.3 \mathrm{pg} / \mathrm{ml}$ vs $83.1 \mathrm{pg} / \mathrm{ml}[\mathrm{p}=0.005]$ and $105.7 \mathrm{pg} / \mathrm{ml}$ vs $89.8 \mathrm{pg} / \mathrm{ml}[\mathrm{p}$ $=0.04]$ for favorable vs unfavorable outcome at 6 and 12 months, respectively). Serum Day 7 GFAP and Day 7 IL-6 levels were significantly higher in unfavorable outcome groups at 6 and 12 months (Day 7 GFAP: $11.9 \mathrm{ng} / \mathrm{ml}$ vs 6.4 $\mathrm{ng} / \mathrm{ml}[\mathrm{p}=0.001]$ and $16.5 \mathrm{ng} / \mathrm{ml}$ vs $6.7 \mathrm{ng} / \mathrm{ml}[\mathrm{p}<0.001]$; and Day 7 IL-6: $75.8 \mathrm{pg} / \mathrm{ml}$ vs $41.1 \mathrm{pg} / \mathrm{ml}[\mathrm{p}=0.004]$; and $90.2 \mathrm{pg} / \mathrm{ml}$ vs $41.3 \mathrm{pg} / \mathrm{ml}[\mathrm{p}=0.001]$ for unfavorable vs favorable outcome at 6 and 12 months, respectively).

\section{Stratified by FIM}

The Eg, Pg, S100-B, IL-6, and TNF- $\alpha$ serum levels were similar across both dichotomized FIM groups at 6 and 12 months ( $p>0.05$ ) (Tables 4 and 5). Serum Day 7 NSE levels were significantly higher in the functionally independent group at 6 months $(20.1 \mathrm{ng} / \mathrm{ml}$ vs $10.5 \mathrm{ng} / \mathrm{ml}$, $\mathrm{p}=0.03$ ). Serum Day 7 GFAP levels were significantly higher in the functionally dependent group at 12 months $(9.7 \mathrm{ng} / \mathrm{ml}$ vs $6.3 \mathrm{ng} / \mathrm{ml}, \mathrm{p}=0.02)$.

\section{Stratified by Survival Status}

The S100-B, NSE, and TNF- $\alpha$ serum levels were similar across both dichotomized survival status groups at 6 and 12 months ( $p>0.05)$ (Tables 6 and 7). At 6 and 12 months, the serum Day $7 \mathrm{Pg}$ and Eg levels at admission were significantly higher in survivors (Day $7 \mathrm{Pg}: 5 \mathrm{ng} / \mathrm{ml}$ vs $1.9 \mathrm{ng} / \mathrm{ml}[\mathrm{p}=0.007]$ and $5.1 \mathrm{ng} / \mathrm{ml}$ vs $1.9 \mathrm{ng} / \mathrm{ml}[\mathrm{p}=$ 0.003 ]; and admission Eg: $0.38 \mathrm{pg} / \mathrm{ml}$ vs $0.14 \mathrm{pg} / \mathrm{ml}[\mathrm{p}=$ $0.03]$ and $0.51 \mathrm{pg} / \mathrm{ml}$ vs $0.14 \mathrm{pg} / \mathrm{ml}[\mathrm{p}=0.05]$ for survivors vs nonsurvivors at 6 and 12 months, respectively). Serum Day 7 GFAP and Day 7 IL-6 levels were significantly higher in nonsurvivors at 6 and 12 months (Day 7 GFAP: $18 \mathrm{ng} / \mathrm{ml}$ vs $6.5 \mathrm{ng} / \mathrm{ml}[\mathrm{p}<0.001]$ and $17.8 \mathrm{ng} / \mathrm{ml}$ vs $6.7 \mathrm{ng} /$ $\mathrm{ml}[\mathrm{p}=0.001]$; Day 7 IL-6: $98.1 \mathrm{pg} / \mathrm{ml}$ vs $41.3 \mathrm{pg} / \mathrm{ml}[\mathrm{p}<$ $0.001]$ and $95.5 \mathrm{pg} / \mathrm{ml}$ vs $41.3 \mathrm{pg} / \mathrm{ml}[\mathrm{p}<0.001]$ for nonsurvivors and survivors at 6 and 12 months, respectively).

\section{Adjusted Binary Logistic Regression Model Analysis}

After adjusting for GCS score and the randomization arm, we observed that Day $7 \mathrm{Pg}$ serum levels had the highest positive odds of predicting favorable GOS score at 6 and 12 months (OR 2.37, CI 1.29-4.36, $\mathrm{p}=0.005$; and OR 3.24, CI 1.5-7, p = 0.003), respectively, and survival at 6 and 12 months (OR 1.9, CI 1.14-3.23, $\mathrm{p}=0.01$; and OR 2 , CI $1.2-3.5, \mathrm{p}=0.01$ ), respectively (Table 8 ). Although the impact occurred to a lesser extent, IL-6 serum levels at admission also positively predicted GOS score at 6 and 12 months (OR 1.03, CI 1.00-1.06, p = 0.02; and OR 1.04, CI $1.00-1.08, \mathrm{p}=0.04)$, respectively. In contrast, Day 7
TABLE 1. Demographic profile and outcome in study population of patients with STBI

\begin{tabular}{|c|c|}
\hline Variable \& Subcategory* & All $(n=86)$ \\
\hline Age† & $32.27 \pm 11.37$ \\
\hline Sex (male) & $73(84.9 \%)$ \\
\hline \multicolumn{2}{|l|}{ Study group } \\
\hline Placebo & $19(22.1 \%)$ \\
\hline $\mathrm{Pg}$ & $20(23.3 \%)$ \\
\hline Hypothermia & $24(27.9 \%)$ \\
\hline Hypothermia + Pg & $23(26.7 \%)$ \\
\hline Average ICP $(\mathrm{mm} \mathrm{Hg}) \dagger$ & $11.81 \pm 4.1$ \\
\hline GCS score at admission $\ddagger$ & $7(6-7)$ \\
\hline Associated injury (polytrauma) & $53(61.6 \%)$ \\
\hline \multicolumn{2}{|l|}{ CT findings } \\
\hline Extradural hematoma & $6(7 \%)$ \\
\hline Subdural hematoma & $30(34.9 \%)$ \\
\hline Fracture & $24(27.9 \%)$ \\
\hline Contusions & $51(59.3 \%)$ \\
\hline $\mathrm{SAH}$ & $24(27.9 \%)$ \\
\hline Infarct & $1(1.2 \%)$ \\
\hline DAl & $11(12.8 \%)$ \\
\hline Cerebral edema & $3(3.5 \%)$ \\
\hline Decompressive craniectomy & $28(32.6 \%)$ \\
\hline Length of hospital stay in days $\ddagger$ & $13(10-18)$ \\
\hline \multicolumn{2}{|c|}{ GOS score at 6 mos-dichotomous $(n=70)$} \\
\hline Unfavorable (1-3) & $20(28.6 \%)$ \\
\hline Favorable (4-5) & $50(71.4 \%)$ \\
\hline GOS score at 6 mos $\ddagger$ & $5(3-5)$ \\
\hline \multicolumn{2}{|l|}{ FIM at 6 mos-dichotomous $(n=60)$} \\
\hline Dependent (18-108) & $22(36.7 \%)$ \\
\hline Independent (109-126) & $38(63.3 \%)$ \\
\hline FIM at 6 mos $\dagger$ & $102.72 \pm 35.41$ \\
\hline \multicolumn{2}{|c|}{ GOS score at 12 mos-dichotomous $(n=65)$} \\
\hline Unfavorable (1-3) & $14(21.5 \%)$ \\
\hline Favorable (4-5) & $51(78.5 \%)$ \\
\hline GOS score at 12 mos $\ddagger$ & $5(4-5)$ \\
\hline \multicolumn{2}{|l|}{ FIM at 12 mos-dichotomous $(n=54)$} \\
\hline Dependent (18-108) & $10(18.5 \%)$ \\
\hline Independent (109-126) & $44(81.5 \%)$ \\
\hline FIM at 12 mos $\dagger$ & $115.48 \pm 25.25$ \\
\hline Mortality rate at 6 mos $(n=70)$ & $10(14.3 \%)$ \\
\hline Mortality rate at $12 \operatorname{mos}(n=65)$ & $11(16.9 \%)$ \\
\hline \multicolumn{2}{|c|}{$\begin{array}{l}\text { SAH = subarachnoid hemorrhage. } \\
\text { * The number of patients in each category varies according to the number } \\
\text { who were either lost to follow-up or dead (in the case of FIM) when the catego- } \\
\text { ries were stratified. } \\
\dagger \text { Values are expressed as the mean } \pm \text { SD. } \\
\neq \text { Values are expressed as the median (IQR). }\end{array}$} \\
\hline
\end{tabular}

GFAP serum levels had the highest negative odds of predicting favorable GOS score at 6 and 12 months (OR 0.85, CI 0.73-0.99, p = 0.04; and OR 0.79, CI 0.65-0.95, p = 0.01 ), respectively, and survival at 6 and 12 months (OR 


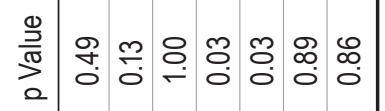
ลิ

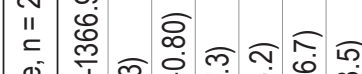

बं

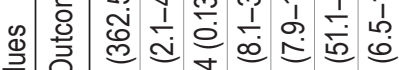
$\frac{\pi}{\pi}$ o

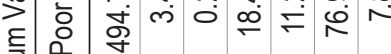

놓

का

莺

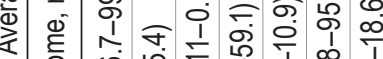

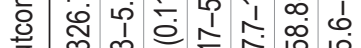

苛

ত ऽ

㞼

즈

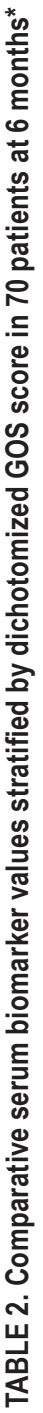

II

๑ 귱

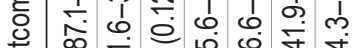

๘

充

足

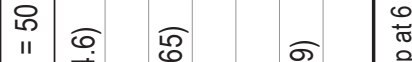

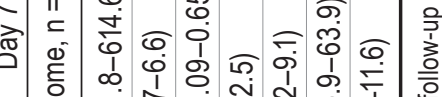

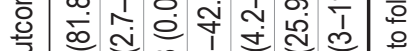

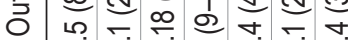

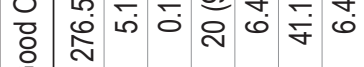
ऽ

祢

ำ

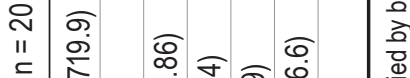

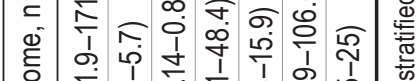

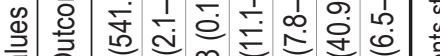

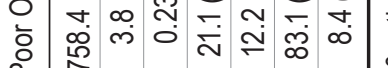

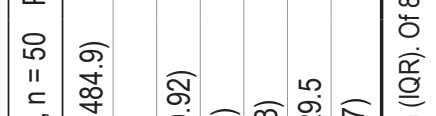

品 过

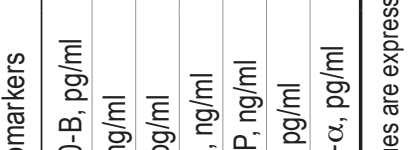

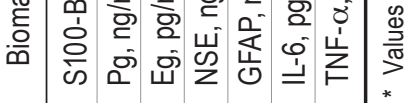

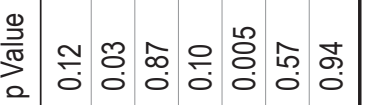

๓

11

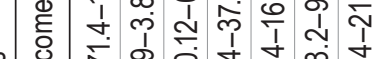

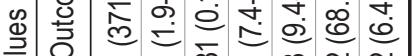

党

닜

क ธิ

弯

离

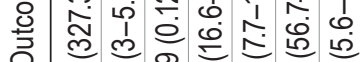

焉

-

蛋

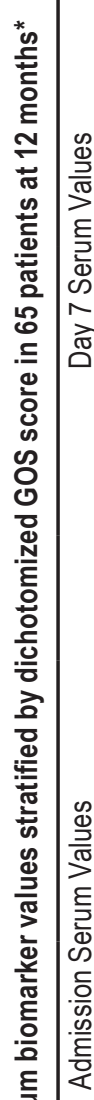

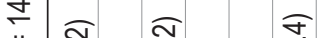

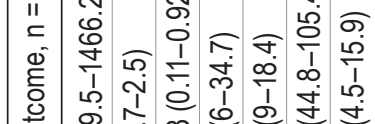

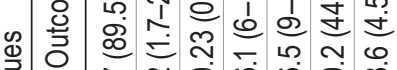

市

in

药 o 0 a

胥 ब

E

矛 这

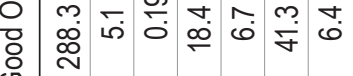

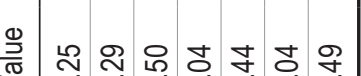

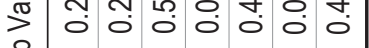

$\forall$ क

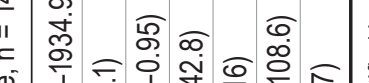

范

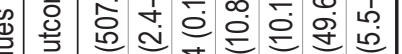

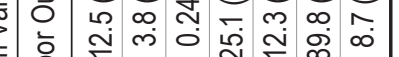

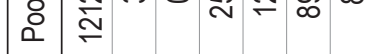

is $\widehat{0}$

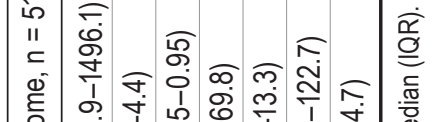

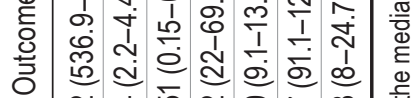

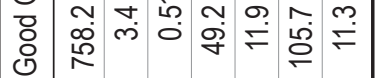

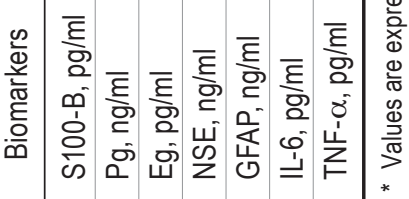

尝

ส

"

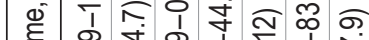

幽 喜

党 인

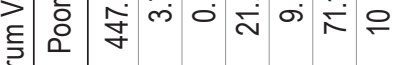

ゆ)

粂

गे

हो

䒓 点

○

ठठ

焉 $\circ$

ส

"I

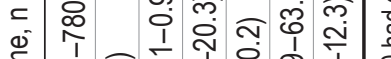

它

क

高它

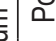

$\infty$

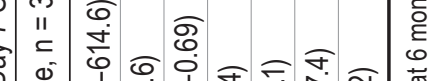

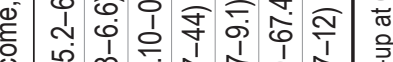

嵌

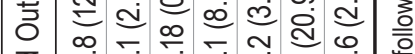

ठ্ট

疍 a 000000000

ส

II İ

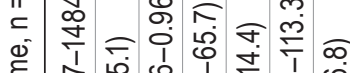

- 1 官 0 L 웅

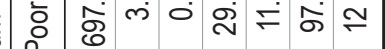

$\infty$

贾

于

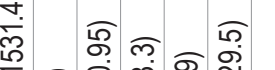

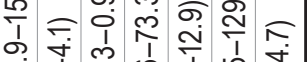

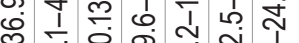

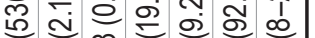

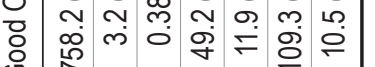

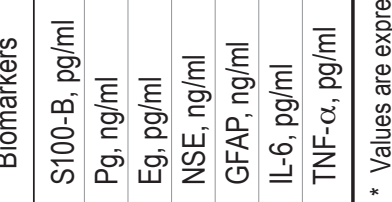



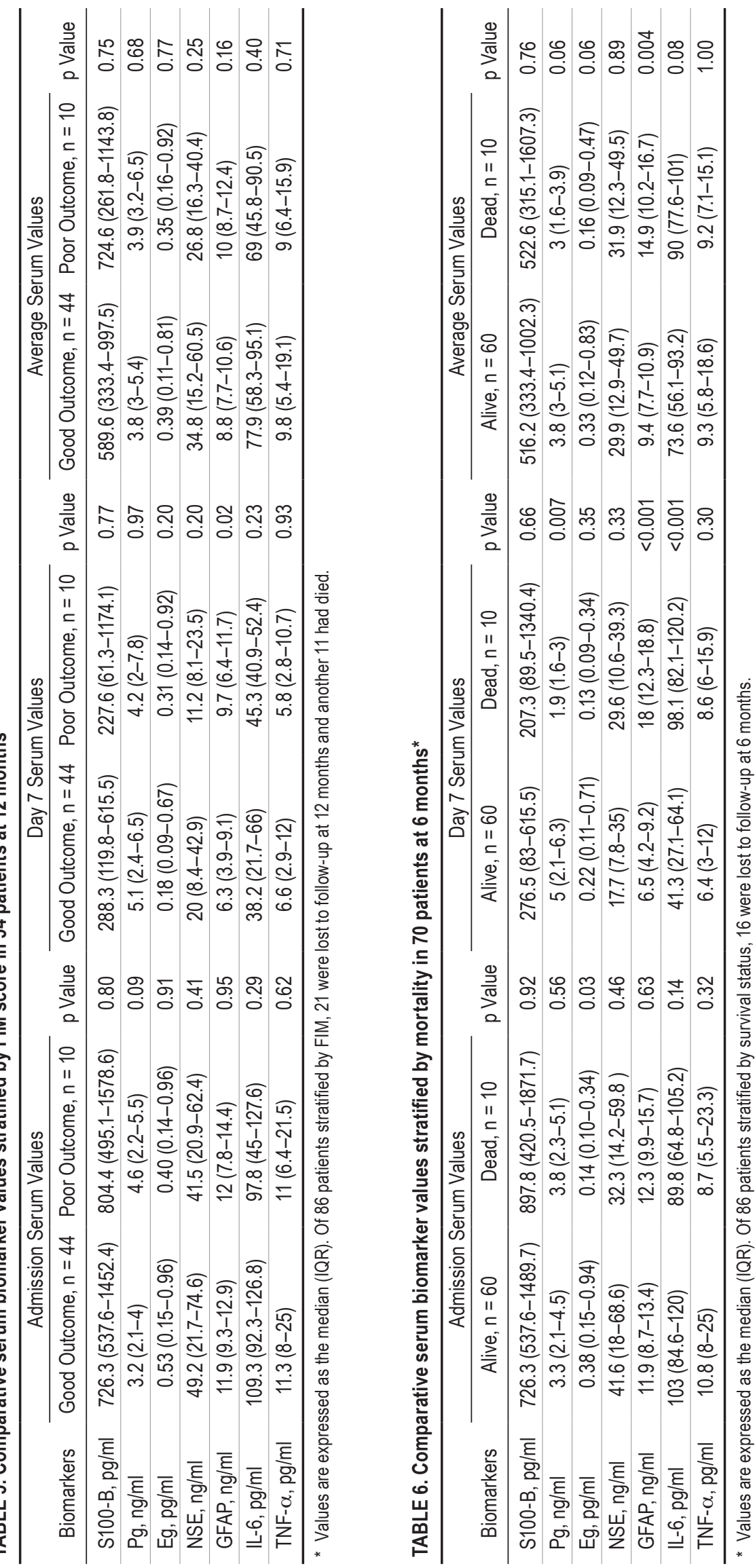

疍

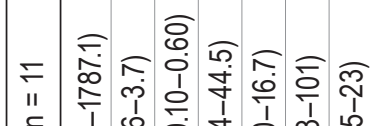

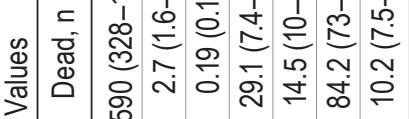

ह

क्षे

总

₹"

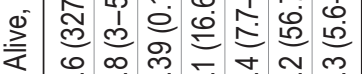

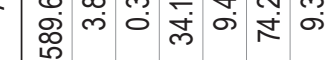

焉

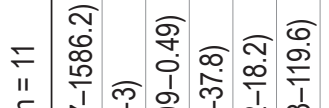

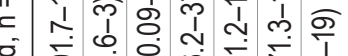

क ष्ठ

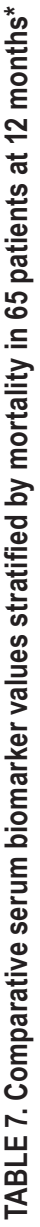

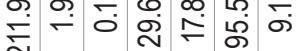

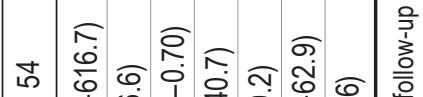
11 1400

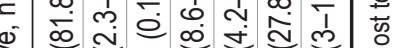

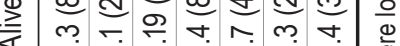
究 is

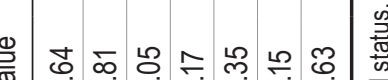
0000000

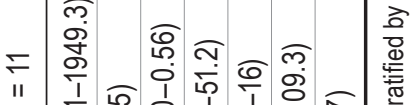

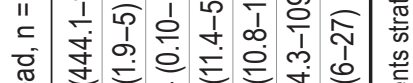

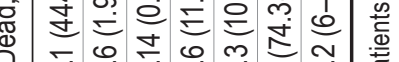

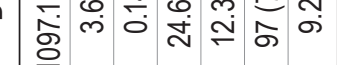

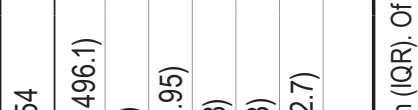

II J) द

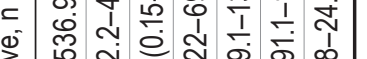

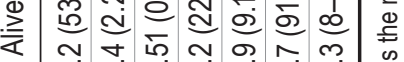

冓

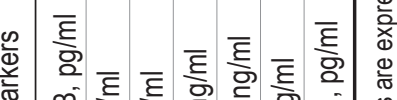

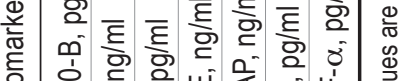

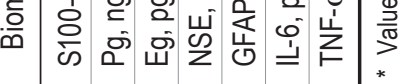


TABLE 8. Adjusted OR for predicting better outcome using a binary logistic multivariate regression model

\begin{tabular}{|c|c|c|c|c|c|}
\hline Outcome & Follow-Up (mos) & Prediction & Parameters & $p$ Value* & OR $(95 \% \mathrm{Cl})$ \\
\hline \multirow[t]{26}{*}{ GOS score (dichotomous) $\dagger$} & \multirow[t]{13}{*}{6} & \multirow[t]{7}{*}{ Favorable } & Pg-D7 & 0.005 & $2.37(1.29-4.36)$ \\
\hline & & & NSE-D1 & 0.86 & $1.00(0.97-1.03)$ \\
\hline & & & IL-6-D1 & 0.02 & $1.03(1.00-1.06)$ \\
\hline & & & GCS score & 0.06 & $1.93(0.98-3.79)$ \\
\hline & & & $\mathrm{Pg}$ & 0.03 & $30.5(1.41-658.4)$ \\
\hline & & & Нypo & 0.08 & $10.8(0.76-154.0)$ \\
\hline & & & Нypo + Pg & 0.06 & $19.4(0.84-450.5)$ \\
\hline & & \multirow[t]{6}{*}{ Unfavorable } & GFAP-D7 & 0.04 & $0.85(0.73-0.99)$ \\
\hline & & & IL-6-D7 & 0.04 & $0.98(0.96-1.00)$ \\
\hline & & & GCS score & 0.03 & $2.01(1.08-3.75)$ \\
\hline & & & $\mathrm{Pg}$ & 0.13 & $4.49(0.63-31.91)$ \\
\hline & & & Нyро & 0.03 & $11.34(1.26-102.3)$ \\
\hline & & & Нypo + Pg & 0.45 & $2.05(0.32-13.06)$ \\
\hline & \multirow[t]{13}{*}{12} & \multirow[t]{7}{*}{ Favorable } & Pg-D7 & 0.003 & $3.24(1.50-6.96)$ \\
\hline & & & NSE-D1 & 0.79 & $1.00(0.97-1.04)$ \\
\hline & & & IL-6-D1 & 0.04 & $1.04(1.00-1.08)$ \\
\hline & & & GCS score & 0.03 & $2.41(1.08-5.41)$ \\
\hline & & & $\mathrm{Pg}$ & 0.25 & $8.64(0.21-349.9)$ \\
\hline & & & Нypo & 0.73 & $1.88(0.05-68.45)$ \\
\hline & & & Нypo + Pg & 0.21 & $9.71(0.27-349.2)$ \\
\hline & & \multirow[t]{6}{*}{ Unfavorable } & GFAP-D7 & 0.01 & $0.79(0.65-0.95)$ \\
\hline & & & IL-6-D7 & 0.06 & $0.98(0.95-1.00)$ \\
\hline & & & GCS score & 0.07 & $1.94(0.93-4.03)$ \\
\hline & & & $\mathrm{Pg}$ & 0.78 & $0.69(0.05-8.83)$ \\
\hline & & & Нyро & 0.62 & $1.99(0.13-30.8)$ \\
\hline & & & Нypo + Pg & 0.76 & $0.68(0.06-7.86)$ \\
\hline \multirow[t]{10}{*}{ FIM† } & \multirow[t]{5}{*}{6} & \multirow[t]{5}{*}{ Favorable } & NSE-D7 & 0.05 & $1.04(1.00-1.08)$ \\
\hline & & & GCS score & 0.003 & 2.39 (1.34-4.27) \\
\hline & & & $\mathrm{Pg}$ & 0.69 & $1.42(0.26-7.81)$ \\
\hline & & & Нyро & 0.39 & $2.15(0.38-12.07)$ \\
\hline & & & Нypo + Pg & 0.40 & $2.41(0.32-18.42)$ \\
\hline & \multirow[t]{5}{*}{12} & \multirow[t]{5}{*}{ Unfavorable } & GFAP-D7 & 0.15 & $0.85(0.68-1.06)$ \\
\hline & & & GCS score & 0.008 & $3.02(1.33-6.85)$ \\
\hline & & & $\mathrm{Pg}$ & 0.21 & $6.62(0.36-122.9)$ \\
\hline & & & Нyро & 0.28 & $3.66(0.35-38.35)$ \\
\hline & & & Нypo + Pg & 0.30 & $4.08(0.28-59.09)$ \\
\hline \multirow[t]{12}{*}{ Survival advantage } & \multirow[t]{6}{*}{6} & \multirow[t]{3}{*}{ Favorable } & $\mathrm{Pg}-\mathrm{D} 7$ & 0.01 & $1.9(1.14-3.23)$ \\
\hline & & & Eg-D1 & 0.09 & $11.78(0.68-205.5)$ \\
\hline & & & GCS score & 0.82 & $1.08(0.56-2.09)$ \\
\hline & & \multirow[t]{3}{*}{ Unfavorable } & GFAP-D7 & 0.01 & $0.64(0.45-0.90)$ \\
\hline & & & IL-6-D7 & 0.006 & $0.96(0.93-0.99)$ \\
\hline & & & GCS score & 0.23 & $0.30(0.04-2.14)$ \\
\hline & \multirow[t]{6}{*}{12} & \multirow[t]{3}{*}{ Favorable } & Pg-D7 & 0.01 & $2(1.17-3.45)$ \\
\hline & & & Eg-D1 & 0.07 & $12.48(0.82-189.65)$ \\
\hline & & & GCS score & 0.19 & $1.57(0.79-3.12)$ \\
\hline & & \multirow[t]{3}{*}{ Unfavorable } & GFAP-D7 & 0.01 & $0.80(0.66-0.96)$ \\
\hline & & & IL-6-D7 & 0.01 & $0.97(0.94-0.99)$ \\
\hline & & & GCS score & 0.81 & $1.11(0.47-2.63)$ \\
\hline
\end{tabular}

D1 = admission value; $D 7$ = Day 7 value; Hypo = hypothermia; Hypo + Pg = combined hypothermia and Pg group.

* A p value $<0.05$ is considered significant.

$\dagger$ Adjusted for both GCS score and randomized treatment group.

$\ddagger$ Adjusted only for GCS score. 
0.64, CI 0.45-0.90, $\mathrm{p}=0.01$; and OR 0.80, CI 0.66-0.96, $\mathrm{p}=0.01$, respectively. Similarly, Day 7 IL-6 serum levels had the highest negative odds of predicting favorable GOS score at 6 months (OR 0.98, CI 0.96-1.00, p = 0.04) and survival at 6 and 12 months (OR 0.96, CI 0.93-0.99, $\mathrm{p}=$ 0.006; and OR 0.97, CI 0.94-0.99, p = 0.01), respectively. Although not statistically significant, there was weak evidence for admission Eg predicting survival ( $p=0.07)$, Day 7 NSE predicting functional independence $(\mathrm{p}=0.05)$, and Day 7 GFAP predicting functional dependence $(p=0.15)$.

\section{The ROC Curve Analysis}

A favorable GOS score (4-5) at 6 and 12 months was predicted by higher admission IL-6 above $108.36 \mathrm{pg} / \mathrm{ml}$ (AUC 0.72, CI 0.59-0.85, sensitivity 53.1\%, and specificity $80 \%$ at 6 months; AUC 0.69, CI $0.54-0.83$, sensitivity $52 \%$, and specificity $78.6 \%$ at 12 months) and Day $7 \mathrm{Pg}$ levels above $3.15 \mathrm{ng} / \mathrm{ml}$ (AUC 0.73, CI 0.60-0.86, sensitivity $69.4 \%$, and specificity $80 \%$ at 6 months; AUC 0.79 , CI $0.68-0.90$, sensitivity $70 \%$, and specificity $92.9 \%$ at 12 months) (Table 9, Figs. 1 and 2). An unfavorable GOS score (1-3) was predicted at 6 and 12 months by higher Day 7 GFAP levels above $9.50 \mathrm{ng} / \mathrm{ml}$ (AUC 0.76, CI 0.61-0.90, sensitivity $60 \%$, and specificity $84 \%$ at 6 months; AUC 0.82 , CI $0.65-0.98$, sensitivity $78.6 \%$, and specificity $82.4 \%$ at 12 months) and by higher Day 7 IL-6 levels above 71.26 $\mathrm{pg} / \mathrm{ml}$ at 6 months (AUC 0.72, CI 0.58-0.86, sensitivity $55 \%$, and specificity $86 \%$ ). Survivors at 6 and 12 months had significantly higher Day $7 \mathrm{Pg}$ levels above $3.15 \mathrm{ng} / \mathrm{ml}$ (AUC 0.77, CI 0.65-0.89, sensitivity 63.3\%, and specificity $90 \%$ at 6 months; AUC 0.78, CI 0.67-0.90, sensitivity $66.7 \%$, and specificity $90.9 \%$ at 12 months). Nonsurvivors at 6 and 12 months had significantly higher Day 7 GFAP serum levels above $11.14 \mathrm{ng} / \mathrm{ml}$ (AUC 0.88, CI 0.71-1.06, sensitivity $90 \%$, and specificity $90 \%$ at 6 months; AUC 0.81 , CI $0.61-1.01$, sensitivity $81.8 \%$, and specificity $88.9 \%$ at 12 months) and Day 7 IL-6 serum levels above $71.26 \mathrm{pg} /$ ml (AUC 0.91, CI 0.83-0.98, sensitivity 90\%, and specificity $85 \%$ at 6 months; AUC 0.87, CI 0.75-0.98, sensitivity $81.8 \%$, and specificity $87 \%$ at 12 months).

\section{Discussion}

\section{Brain Tissue Antigens}

The neuronal injury marker NSE is an isoenzyme of enolase ( $\mathrm{t}_{1 / 2}$ approximately 48 hours) that is present predominantly in the cytoplasm of neurons, has poor correlation with contusion volume, and is essentially a marker of diffuse axonal injury (DAI).,16,17,19,20 However, its specificity for neurons is limited by confounding factors like sepsis, hypoperfusion, extracranial trauma, bleeding, and liver or kidney damage, in which serum NSE level also increases, making it of less clinical relevance in patients with polytrauma.,20 The NSE levels in the first 72 hours postinjury have been more closely related with outcome, ${ }^{21}$ probably because of its short half-life. However, in the present cohort with the high incidence of polytrauma (62\%) and low incidence of DAI (13\%), the relevance of serum NSE levels in the present context is miniscule. We also failed to observe any statistically significant impact of NSE levels on outcome in the adjusted binary logistic model. More specific neuronal markers like ubiquitin Cterminal hydrolase L1 have shown more consistent results in recent trials. ${ }^{3,4}$

$\mathrm{A} \mathrm{Ca}^{2+}$-binding protein ( $\mathrm{t}_{1 / 2}$ approximately 2 hours) predominantly secreted by astrocytes, S100-B is correlated with contusion volume. Despite a few studies showing serum $\mathrm{S100-B}$ as a predictor of outcome, its serum assay is not ideal because of its limited blood-brain barrier (BBB)

TABLE 9. The ROC curve analysis

\begin{tabular}{|c|c|c|c|c|c|}
\hline Outcome & Serum Biomarker & Cutoff Value* & AUC $(95 \% \mathrm{Cl}) \dagger$ & Sensitivity (\%) & Specificity (\%) \\
\hline \multicolumn{6}{|l|}{ GOS score (dichotomous) } \\
\hline \multirow[t]{2}{*}{ Favorable (6 mos) } & Pg-D7, ng/ml & 3.15 & $0.73(0.60-0.86)$ & 69.4 & 80 \\
\hline & IL-6-D1, pg/ml & 108.36 & $0.72(0.59-0.85)$ & 53.1 & 80 \\
\hline \multirow[t]{2}{*}{ Favorable (12 mos) } & Pg-D7, ng/ml & 3.15 & $0.79(0.68-0.90)$ & 70 & 92.9 \\
\hline & IL-6-D1, pg/ml & 108.36 & $0.69(0.54-0.83)$ & 52 & 78.6 \\
\hline \multicolumn{6}{|l|}{ GOS score (dichotomous) } \\
\hline \multirow[t]{2}{*}{ Unfavorable (6 mos) } & GFAP-D7, ng/ml & 9.50 & $0.76(0.61-0.90)$ & 60 & 84 \\
\hline & IL-6-D7, pg/ml & 71.26 & $0.72(0.58-0.86)$ & 55 & 86 \\
\hline Unfavorable (12 mos) & GFAP-D7, ng/ml & 9.50 & $0.82(0.65-0.98)$ & 78.6 & 82.4 \\
\hline \multicolumn{6}{|l|}{ Survivors } \\
\hline 6 mos & $\mathrm{Pg}-\mathrm{D} 7, \mathrm{ng} / \mathrm{ml}$ & 3.15 & $0.77(0.65-0.89)$ & 63.3 & 90 \\
\hline 12 mos & $\mathrm{Pg}-\mathrm{D} 7, \mathrm{ng} / \mathrm{ml}$ & 3.15 & $0.78(0.67-0.90)$ & 66.7 & 90.9 \\
\hline \multicolumn{6}{|l|}{ Nonsurvivors } \\
\hline \multirow[t]{2}{*}{$6 \mathrm{mos}$} & GFAP-D7, ng/ml & 11.14 & $0.88(0.71-1.06)$ & 90 & 90 \\
\hline & IL-6-D7, pg/ml & 71.26 & $0.91(0.83-0.98)$ & 90 & 85 \\
\hline \multirow[t]{2}{*}{12 mos } & GFAP-D7, ng/ml & 11.14 & $0.81(0.61-1.01)$ & 81.8 & 88.9 \\
\hline & IL-6-D7, pg/ml & 71.26 & $0.87(0.75-0.98)$ & 81.8 & 87 \\
\hline
\end{tabular}

* Cutoff values were calculated using the Youden index.

$\dagger$ Area under the curve $(95 \% \mathrm{Cl})$ representing accuracy of the test performed. 

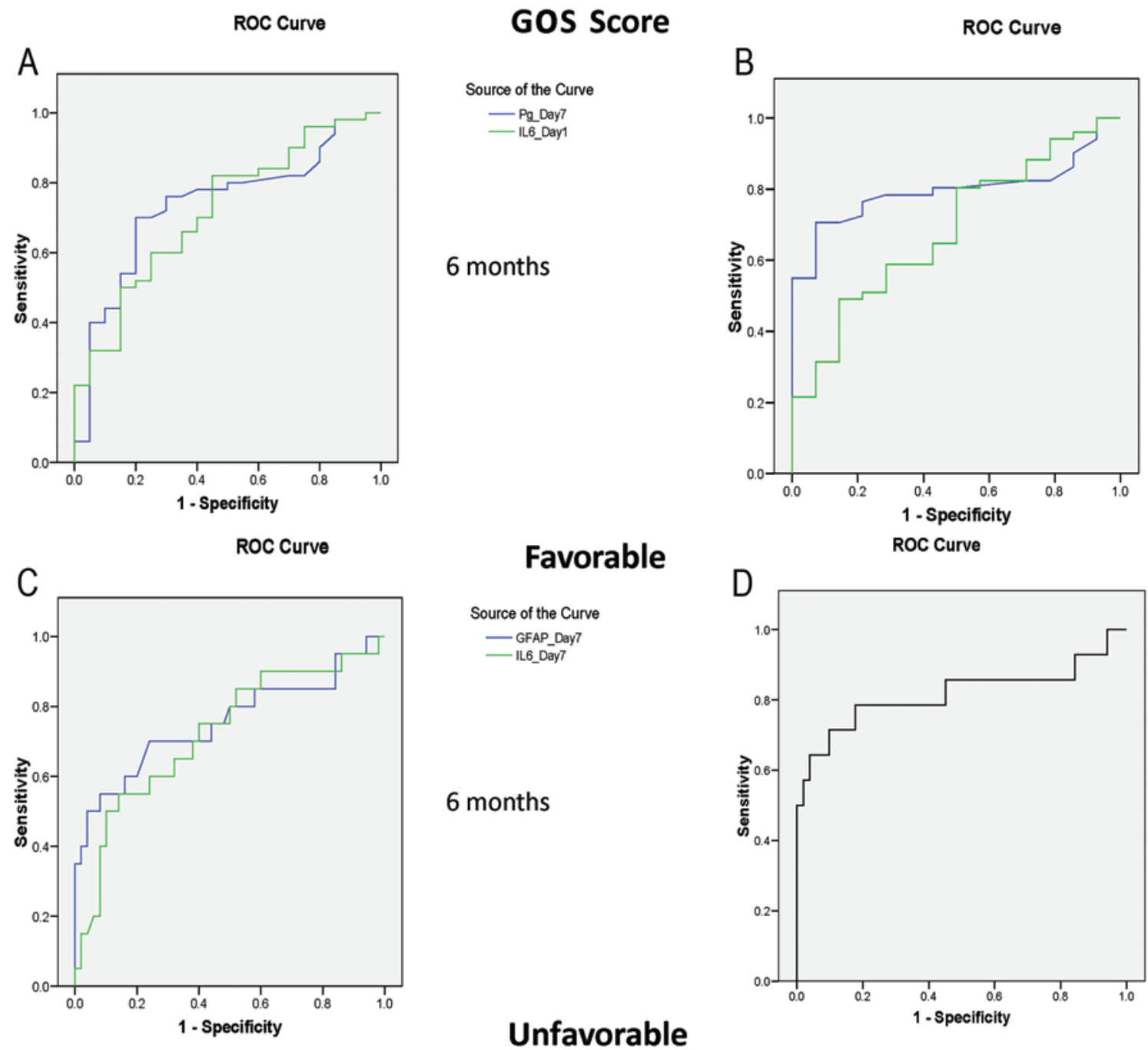
Source of the Curve - Pg_Day7 - IL_Day1

\section{2 months}

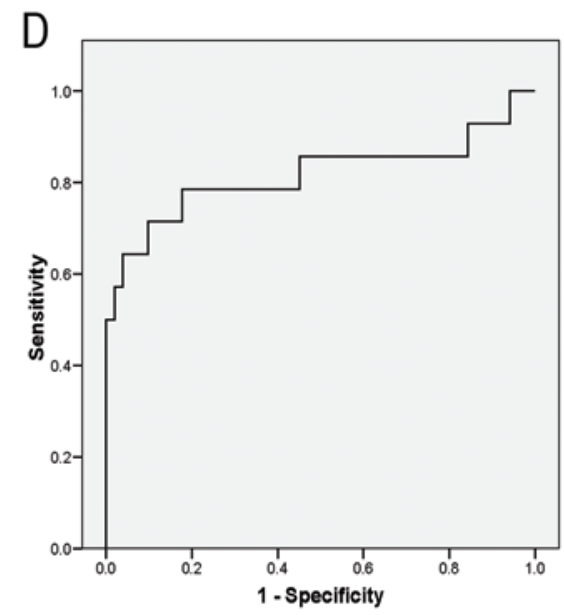

Source of the Curve --GFAP_Day7

12 months

FIG. 1. The ROC curve for truly independent factors predicting favorable and unfavorable GOS score at 6 and 12 months. Figure is available in color online only.

permeability, short half-life (small window for sampling), and peripheral source of production (besides CNS). ${ }^{10,18,28}$ Cerebrospinal fluid or brain tissue S100-B estimation is a better predictor of outcome. We failed to observe any association between serum S100-B and any of the outcome parameters, probably because of the above-mentioned factors.

Glial fibrillary acidic protein, an intermediate filament in astrocyte cytoskeleton ( $\mathrm{t}_{1 / 2}$ approximately 48 hours), is a much more specific marker of glial injury and BBB disruption with good discriminative value for focal mass lesions (contusion), and is an independent predictor of poor outcome. . $^{3,4,16,17,28}$ We observed statistically significant odds of predicting poor outcome at long-term follow-up (1 year) by using serum Day 7 GFAP levels above $9.50 \mathrm{ng} / \mathrm{ml}$ with high accuracy (AUC 0.82) and specificity (82.4\%). Serum Day 7 GFAP levels above $11.14 \mathrm{ng} / \mathrm{ml}$ predicted long-term mortality (1 year) with high accuracy (AUC 0.81) and specificity (88.9\%). Because GFAP has a short half-life, we hypothesize that delayed (Day 7) estimation of serum
GFAP levels could be more beneficial than the initial estimation at admission for detecting patients at long-term risk; it is an estimation of ongoing secondary neuronal injury occurring after 5-7 days, thereby causing elevation of GFAP levels again and emphasizing the role of additional measures to optimize the estimation. Because all of these antigens have relatively short half-lives, identification and prognostication in subacute and chronic TBI models is not possible. Recently, a trial assessing the role of IgG antiGFAP antibody, which can be estimated from Day 7 until 6 months postinjury, has shown it to be a marker of BBB disruption and inversely correlated with outcome. ${ }^{37}$

\section{Cytokines}

Tumor necrosis factor- $\alpha$ is a potent proinflammatory cytokine, produced primarily by microglia and astrocytes in CNS as a result of combined DAI and hypoxia, with a conflicting dual profile of its impact on outcome data. ${ }^{13,22,32}$ Its concentration in brain parenchyma increases within 1 hour after TBI and peaks between 4 and 8 hours. ${ }^{32}$ For 

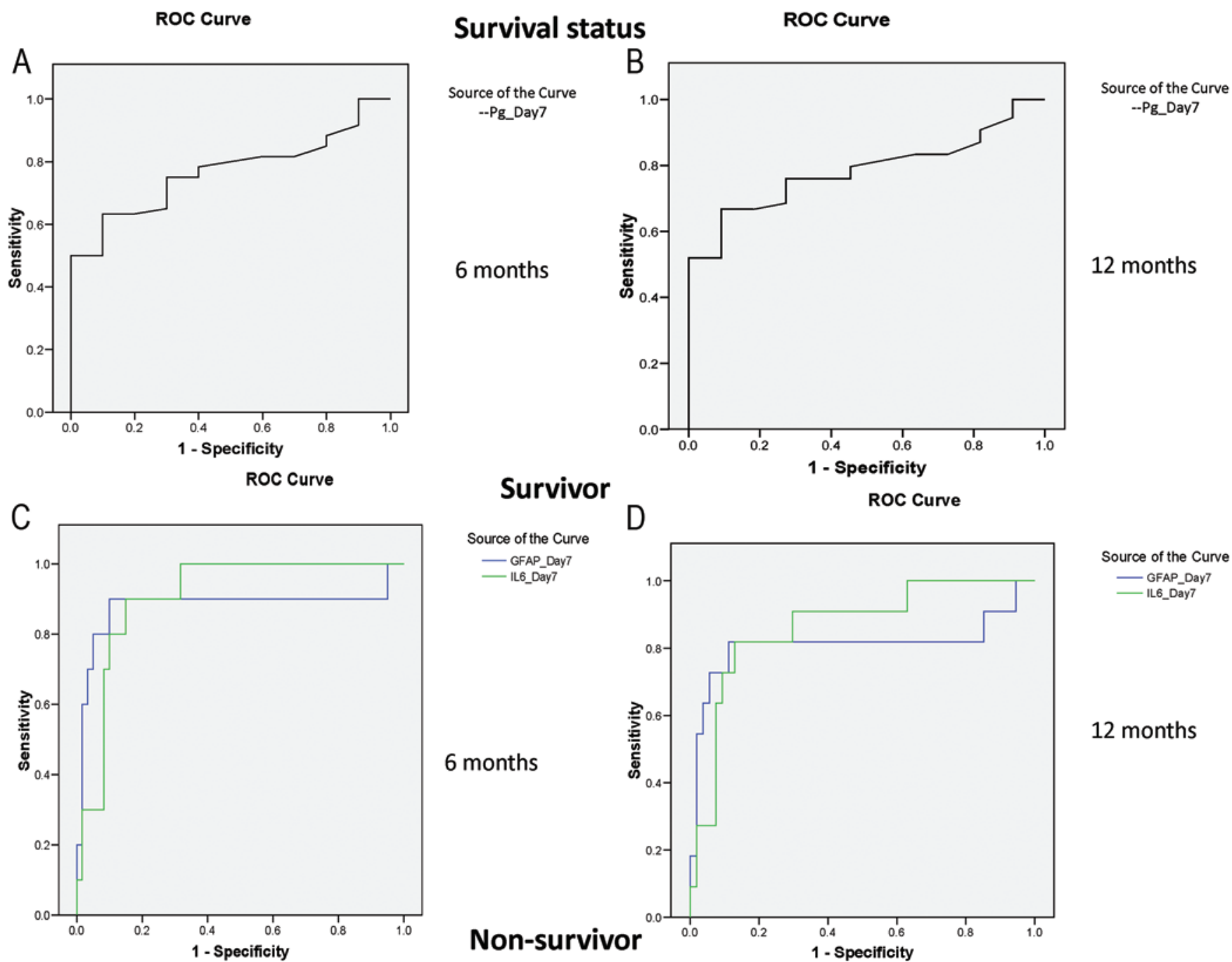

FIG. 2. The ROC curve for truly independent factors predicting survivors and nonsurvivors at 6 and 12 months. Figure is available in color online only.

clinical use, its concentration in CSF is a more reliable indicator (peaks at approximately 24 hours) than serum, because it excludes elevations due to polytrauma. ${ }^{32}$ Serum TNF- $\alpha$ concentration correlates moderately with rise in ICP, providing a window of opportunity to intervene before secondary brain damage. ${ }^{26}$ We failed to identify any significant association between the serum levels of TNF$\alpha$ and either of the outcome variables, probably because of confounding by a high incidence of polytrauma $(62 \%)$ and a low incidence of DAI (13\%), making an appropriate inference difficult.

Interleukin-6 is another cytokine expressed in CNS (microglia, astrocytes, and neurons), again with conflicting data in the existing literature regarding its role as an independent predictor of ICP, mortality, and outcome. . $^{1,2,6,8,9,11-13,30,32}$ Similar to TNF- $\alpha$, it is detectable by 1 hour postinjury and peaks in brain parenchyma at approximately $2-8$ hours..$^{32}$ However, unlike TNF- $\alpha$, particularly related to the DAI model, IL-6 levels do not depend on the injury model, and this is a more sensitive marker. ${ }^{32}$ It inhibits TNF- $\alpha$ synthesis and $N$-methyl-D-aspartate-mediated toxicity, and it induces nerve growth factor (NGF) and promotes neural differentiation and survival. ${ }^{32}$ Despite representing BBB dysfunction and more reliable serum concentrations than TNF- $\alpha$, it is still less specific and partly affected by the addition of polytrauma to the TBI model. For increasing specificity in the TBI model, recent studies have incorporated the NGF:IL-6 ratio as a more specific predictor and have also included cerebral microdialysis for the most accurate estimation of ongoing variation of cytokines in close vicinity to the actual lesion. ${ }^{32}$

We observed statistically significant odds of predicting poor outcome at long-term follow-up (6 months) by using serum Day 7 IL-6 levels above $71.26 \mathrm{pg} / \mathrm{ml}$, with adequate accuracy (AUC 0.72) and specificity (86\%). Serum Day 7 IL-6 levels above $71.26 \mathrm{pg} / \mathrm{ml}$ predicted long-term mortality (1 year) with high accuracy (AUC 0.87) and specificity $(87 \%)$. These observations were in line with the delayed rise in serum GFAP levels, predicting long-term outcome and mortality, representing a dismal outcome due to sec- 
ondary brain damage. Paradoxically, an elevated serum IL-6 concentration above $108.36 \mathrm{pg} / \mathrm{ml}$ at admission $(<8$ hours postinjury) was found to be predictive of long-term (1 year) favorable outcome, although with a poor accuracy (AUC 0.69) and lower specificity (78.6\%). It is presumed that elevation in these proinflammatory cytokines is an adaptive response of the brain to injury, which causes transient destruction and apoptosis of damaged neural cells, paving the way for the reparative process. Therefore we hypothesize that an initial elevation of IL-6 levels could be protective in the long run, whereas a delayed rise could be catastrophic because it usually represents more grave conditions like elevated ICP, sepsis, multiorgan dysfunction syndrome, and shock.

\section{Gonadal Hormones}

Estradiol offers neuroprotection by ameliorating excitotoxicity, promoting neuronal lactate utilization, maintaining cerebral blood flow, and decreasing apoptosis in TBI models. 5, ${ }^{15,23,25,29}$ However, this presumed benefit has not been consistently translated from animal (rodent) models to patients in clinical trials, partly due to a difference between the two groups in production of Eg via peripheral aromatization, which is selectively present in primates only. ${ }^{5,15,23,25,29} \mathrm{Up}$ to $80 \%$ of patients with sTBI have suppression of the hypothalamic-pituitary-gonadal axis and activation of stress hormones produced by the adrenal gland; therefore, $\mathrm{Eg}$ production is predominantly under the control of peripheral aromatization in adipose tissue, which in turn is positively affected by proinflammatory cytokines like TNF- $\alpha .{ }^{29}$ Because there is a surge of systemic proinflammatory chemokines immediately following sTBI, the injury increases Eg levels as a byproduct of ongoing inflammatory response, thereby reflecting a global illness rather than a causal role. ${ }^{5,29}$ Few studies have shown a capacity of serum Eg levels at 48 hours to predict mortality, ${ }^{5,15}$ but their model failed to incorporate proinflammatory cytokines, a major confounder in the primate TBI model. As expected, we failed to observe any statistically significant impact of admission Eg levels on mortality in the adjusted binary logistic model, despite a weak association evident with survival status ( $p=0.09$ and $\mathrm{p}=0.07$ at 6 and 12 months, respectively).

Progesterone mediates its neuroprotection by reducing cerebral edema, lipid peroxidation, isoprostanes, and expression of proinflammatory genes; generating metabolites that reduce proapoptotic and increase antiapoptotic enzymes; and modifying the expression of vascular endothelial growth factor, brain-derived neurotrophic growth factor, and aquaporins responsible for development of edema. ${ }^{14}$ Besides these direct effects, it also indirectly affects the TBI model by modulating Eg, testosterone, and cortisol levels. ${ }^{29}$ Although previous Phase II trials confirmed a beneficial role of Pg therapy in an STBI model for optimal outcome, ${ }^{33,35}$ more recent Phase III clinical trials failed to do so. ${ }^{24,34}$ We observed statistically significant odds of predicting favorable outcome at long-term follow-up (1 year) using serum Day $7 \mathrm{Pg}$ levels above $3.15 \mathrm{ng} / \mathrm{ml}$ with adequate accuracy (AUC 0.79) and high specificity (92.9\%). Serum Day $7 \mathrm{Pg}$ levels above $3.15 \mathrm{ng} / \mathrm{ml}$ also predicted survivors with adequate accuracy (AUC 0.78) and high specificity (90.9\%). These results imply the importance of maintaining serum levels of Pg over $3.15 \mathrm{ng} / \mathrm{ml}$ until 7 days postinjury for optimal outcome.

\section{Limitations of the Study}

Small sample size, lack of sampling data between admission and Day 7, and a higher percentage of patients lost to follow-up are the primary limitations of this study. Inclusion of only adults with sTBI might not allow generalization of our results to all TBI models. Lack of data on body mass index and menopausal status may have affected the analysis of effects of gonadal hormones.

\section{Conclusions}

Serial monitoring and optimization of serum Pg, GFAP, and IL-6 levels could aid in prognostication in patients with sTBI and guide us to direct more resources toward such patients for optimal outcome. A cause and effect relationship or a mere association of these biomarkers to outcome needs to be further studied for better understanding of the pathophysiology in sTBI and for choosing potential therapeutic targets. Ultimately, a multidimensional prognostic model that incorporates the biochemical profile along with clinical and radiological parameters will be useful for clinical practice as well as clinical research.

\section{Acknowledgments}

This study was funded by the Department of Biotechnology, Ministry of Science and Technology, India.

\section{References}

1. Arand M, Melzner H, Kinzl L, Brückner UB, Gebhard F: Early inflammatory mediator response following isolated traumatic brain injury and other major trauma in humans. Langenbecks Arch Surg 386:241-248, 2001

2. Chiaretti A, Antonelli A, Mastrangelo A, Pezzotti P, Tortorolo L, Tosi F, et al: Interleukin-6 and nerve growth factor upregulation correlates with improved outcome in children with severe traumatic brain injury. J Neurotrauma 25:225-234, 2008

3. Czeiter E, Mondello S, Kovacs N, Sandor J, Gabrielli A, Schmid K, et al: Brain injury biomarkers may improve the predictive power of the IMPACT outcome calculator. J Neurotrauma 29:1770-1778, 2012

4. Diaz-Arrastia R, Wang KK, Papa L, Sorani MD, Yue JK, Puccio AM, et al: Acute biomarkers of traumatic brain injury: relationship between plasma levels of ubiquitin Cterminal hydrolase-L1 and glial fibrillary acidic protein. J Neurotrauma 31:19-25, 2014

5. Dossett LA, Swenson BR, Evans HL, Bonatti H, Sawyer RG, May AK: Serum estradiol concentration as a predictor of death in critically ill and injured adults. Surg Infect (Larchmt) 9:41-48, 2008

6. Ferreira LC, Regner A, Miotto KD, Moura Sd, Ikuta N, Vargas AE, et al: Increased levels of interleukin-6, -8 and -10 are associated with fatal outcome following severe traumatic brain injury. Brain Inj 28:1311-1316, 2014

7. Guzel A, Er U, Tatli M, Aluclu U, Ozkan U, Duzenli Y, et al: Serum neuron-specific enolase as a predictor of short-term outcome and its correlation with Glasgow Coma Scale in traumatic brain injury. Neurosurg Rev 31:439-445, 2008

8. Hergenroeder GW, Moore AN, McCoy JP Jr, Samsel L, Ward NH III, Clifton GL, et al: Serum IL-6: a candidate biomarker 
for intracranial pressure elevation following isolated traumatic brain injury. J Neuroinflammation 7:19, 2010

9. Kalabalikis P, Papazoglou K, Gouriotis D, Papadopoulos N, Kardara M, Papageorgiou F, et al: Correlation between serum IL-6 and CRP levels and severity of head injury in children. Intensive Care Med 25:288-292, 1999

10. Kleindienst A, Ross Bullock M: A critical analysis of the role of the neurotrophic protein S100B in acute brain injury. $\mathbf{J}$ Neurotrauma 23:1185-1200, 2006

11. Kossmann T, Hans V, Imhof HG, Trentz O, Morganti-Kossmann MC: Interleukin-6 released in human cerebrospinal fluid following traumatic brain injury may trigger nerve growth factor production in astrocytes. Brain Res 713:143-152, 1996

12. Kumar RG, Diamond ML, Boles JA, Berger RP, Tisherman SA, Kochanek PM, et al: Acute CSF interleukin-6 trajectories after TBI: associations with neuroinflammation, polytrauma, and outcome. Brain Behav Immun 45:253-262, 2015

13. Lenzlinger PM, Morganti-Kossmann MC, Laurer HL, McIntosh TK: The duality of the inflammatory response to traumatic brain injury. Mol Neurobiol 24:169-181, 2001

14. Ma J, Huang S, Qin S, You C: Progesterone for acute traumatic brain injury. Cochrane Database Syst Rev 10:CD008409, 2012

15. May AK, Dossett LA, Norris PR, Hansen EN, Dorsett RC, Popovsky KA, et al: Estradiol is associated with mortality in critically ill trauma and surgical patients. Crit Care Med 36:62-68, 2008

16. Mondello S, Jeromin A, Buki A, Bullock R, Czeiter E, Kovacs $\mathrm{N}$, et al: Glial neuronal ratio: a novel index for differentiating injury type in patients with severe traumatic brain injury. J Neurotrauma 29:1096-1104, 2012

17. Mondello S, Papa L, Buki A, Bullock MR, Czeiter E, Tortella FC, et al: Neuronal and glial markers are differently associated with computed tomography findings and outcome in patients with severe traumatic brain injury: a case control study. Crit Care 15:R156, 2011

18. Nguyen DN, Spapen H, Su F, Schiettecatte J, Shi L, HachimiIdrissi S, et al: Elevated serum levels of S-100beta protein and neuron-specific enolase are associated with brain injury in patients with severe sepsis and septic shock. Crit Care Med 34:1967-1974, 2006

19. Ogata M, Tsuganezawa O: Neuron-specific enolase as an effective immunohistochemical marker for injured axons after fatal brain injury. Int J Legal Med 113:19-25, 1999

20. Pelinka LE, Hertz H, Mauritz W, Harada N, Jafarmadar M, Albrecht M, et al: Nonspecific increase of systemic neuronspecific enolase after trauma: clinical and experimental findings. Shock 24:119-123, 2005

21. Schaarschmidt H, Prange HW, Reiber H: Neuron-specific enolase concentrations in blood as a prognostic parameter in cerebrovascular diseases. Stroke 25:558-565, 1994

22. Shohami E, Ginis I, Hallenbeck JM: Dual role of tumor necrosis factor alpha in brain injury. Cytokine Growth Factor Rev 10:119-130, 1999

23. Simpson ER, Ackerman GE, Smith ME, Mendelson CR: Estrogen formation in stromal cells of adipose tissue of women: induction by glucocorticosteroids. Proc Natl Acad Sci U S A 78:5690-5694, 1981

24. Skolnick BE, Maas AI, Narayan RK, van der Hoop RG, MacAllister T, Ward JD, et al: A clinical trial of progesterone for severe traumatic brain injury. N Engl J Med 371:2467-2476, 2014

25. Stein DG: Brain damage, sex hormones and recovery: a new role for progesterone and estrogen? Trends Neurosci 24:386-391, 2001

26. Stein DM, Lindel AL, Murdock KR, Kufera JA, Menaker J, Scalea TM: Use of serum biomarkers to predict secondary insults following severe traumatic brain injury. Shock 37:563-568, 2012
27. Stein DM, Lindell A, Murdock KR, Kufera JA, Menaker J, Keledjian K, et al: Relationship of serum and cerebrospinal fluid biomarkers with intracranial hypertension and cerebral hypoperfusion after severe traumatic brain injury. J Trauma 70:1096-1103, 2011

28. Vos PE, Jacobs B, Andriessen TM, Lamers KJ, Borm GF, Beems T, et al: GFAP and S100B are biomarkers of traumatic brain injury: an observational cohort study. Neurology 75:1786-1793, 2010

29. Wagner AK, McCullough EH, Niyonkuru C, Ozawa H, Loucks TL, Dobos JA, et al: Acute serum hormone levels: characterization and prognosis after severe traumatic brain injury. J Neurotrauma 28:871-888, 2011

30. Winter CD, Pringle AK, Clough GF, Church MK: Raised parenchymal interleukin-6 levels correlate with improved outcome after traumatic brain injury. Brain 127:315-320, 2004

31. Wolf H, Frantal S, Pajenda GS, Salameh O, Widhalm H, Hajdu S, et al: Predictive value of neuromarkers supported by a set of clinical criteria in patients with mild traumatic brain injury: S100B protein and neuron-specific enolase on trial: clinical article. J Neurosurg 118:1298-1303, 2013

32. Woodcock T, Morganti-Kossmann MC: The role of markers of inflammation in traumatic brain injury. Front Neurol 4:18, 2013

33. Wright DW, Kellermann AL, Hertzberg VS, Clark PL, Frankel M, Goldstein FC, et al: ProTECT: a randomized clinical trial of progesterone for acute traumatic brain injury. Ann Emerg Med 49:391-402, 402.e1-402.e2, 2007

34. Wright DW, Yeatts SD, Silbergleit R, Palesch YY, Hertzberg VS, Frankel M, et al: Very early administration of progesterone for acute traumatic brain injury. N Engl J Med 371:2457-2466, 2014

35. Xiao G, Wei J, Yan W, Wang W, Lu Z: Improved outcomes from the administration of progesterone for patients with acute severe traumatic brain injury: a randomized controlled trial. Crit Care 12:R61, 2008

36. Yan EB, Hellewell SC, Bellander BM, Agyapomaa DA, Morganti-Kossmann MC: Post-traumatic hypoxia exacerbates neurological deficit, neuroinflammation and cerebral metabolism in rats with diffuse traumatic brain injury. $\mathbf{J}$ Neuroinflammation 8:147, 2011

37. Zhang Z, Zoltewicz JS, Mondello S, Newsom KJ, Yang Z, Yang B, et al: Human traumatic brain injury induces autoantibody response against glial fibrillary acidic protein and its breakdown products. PLoS One 9:e92698, 2014

\section{Disclosures}

The authors report no conflict of interest concerning the materials or methods used in this study or the findings specified in this paper.

\section{Author Contributions}

Conception and design: Sinha. Acquisition of data: Samson. Analysis and interpretation of data: Raheja. Drafting the article: Raheja, Samson. Critically revising the article: Sinha, Bhoi, Subramanian. Reviewed submitted version of manuscript: all authors. Approved the final version of the manuscript on behalf of all authors: Sinha. Statistical analysis: Raheja. Administrative/technical/material support: Sinha, Bhoi, Subramanian, P Sharma. Study supervision: Sinha, BS Sharma.

\section{Correspondence}

Sumit Sinha, Department of Neurosurgery and Gamma Knife, Neurosciences Centre, F25, Aiims Campus, Ansary Nagar, All India Institute of Medical Sciences, New Delhi 110029, India. email: sumitneuro@gmail.com. 